Anuario Latinoamericano Ciencias Políticas

y Relaciones Internacionales

vol. 8, 2019

pp. $173-188$

\section{Las políticas de género durante la presidencia de Cristina Fernández de Kirchner en Argentina (2007-2015)}

DOI: 10.17951/al.2019.8.173-188

\section{Gender Policies During the Presidency of Cristina Fernández de Kirchner in Argentina (2007-2015)}

\author{
Magdalena Lisińska ${ }^{*}$ \\ UNIVERSIDAD JAGUELÓNICA, CRACOVIA, POLONIA \\ $\triangle$ m.lisinska@gmail.com \\ https://orcid.org/0000-0001-9625-9495
}

\title{
RESUMEN:
}

El objetivo principal del presente artículo es discutir las políticas de género -propuestas y agendas, legislación e iniciativas políticas durante la presidencia de Cristina Fernández de Kirchner en Argentina (2007-2015). Primero, analizo los éxitos de la presidencia de Cristina Fernández - en la legislación y en las cuestiones de la violencia de género. Luego, presento los problemas y las derrotas de la agenda kirchnerista en el ámbito de género. En la conclusión, resumo los argumentos presentados en el artículo y considero si la presidencia de Cristina Fernández de Kirchner puede ser identificada como un paso adelante en materia de la igualdad de géneros, los derechos igualitarios y el empoderamiento de las mujeres en Argentina.

PALABRAS CLAVE: Argentina, Cristina Fernández de Kirchner, género, igualdad de género, políticas de género, políticas igualitarias, kirchnerismo.

\section{ABSTRACT:}

The main objective of this article is to discuss gender policies - proposals and agendas, legislation and political initiatives during the presidency of Cristina Fernández de Kirchner in Argentina (2007-2015). Firstly, I analyze the achievements of Kirchner's presidency on the discussed field - in legislation and in the fight against gender violence. Subsequently, I present failures and problems of the Kirchner's agenda. In the conclusions I summarize the arguments presented in the article and consider whether the presidency of Cristina Fernández de Kirchner can be identified as

* Doctora en Ciencia Política por la Universidad Jaguelónica, Cracovia, Polonia. Sus líneas de investigación son la política e historia de Argentina, con un enfoque en la última dictadura militar 1976-1983 y las relaciones entre los Estados Unidos y América Latina. 
Dossier América Latina: género y política a step forward in terms of gender equality, women's empowerment and equal rights in Argentina.

KEYWORDS: Argentina, Cristina Fernández de Kirchner, gender, gender equality, gender politics, egalitarian Policies, Kirchnerism.

\section{Introducción}

Cristina Fernández de Kirchner fue la segunda mujer presidente en la historia de Argentina y la primera elegida en las elecciones generales. A pesar de que asumió la función inmediatamente después de su marido, el presidente Néstor Kirchner (2003-2007), sus ambiciones políticas habían sido evidentes incluso antes. Tras su carrera como diputada y senadora en el Congreso de la Nación Argentina, Fernández de Kirchner logró desarrollar su propia posición política ${ }^{1}$. Incluso, en la víspera de las elecciones presidenciales de 2003, Cristina había tenido una trayectoria política de mayor impacto nacional que su esposo, el gobernador de la provincia sureña de Santa Cruz. No obstante, le apoyaba durante su campaña y presidencia, finalmente aceptando la nominación para ser su sucesora en las elecciones del 2007. Fernández de Kirchner ganó en primera vuelta por el 45,28 \% de los votos (Dirección Nacional Electoral, 2007). Teniendo a Evita Perón como la figura de referencia, Cristina despertó muchas esperanzas incluso durante su campaña, dirigiéndose directamente a las mujeres y hablando sobre las mujeres. Como lo explican Vitale y Maizels (2011, pp. 350-351), Cristina trataba de construir "el ethos de la feminidad" y así generar identificación con las mujeres argentinas. Su elección generó grandes expectativas, especialmente entre los que esperaban una nueva legislación igualitaria de género.

Cabe señalar que el género, también conocido como el sexo cultural, es una categoría social. Tiene su base en las diferencias entre hombres y mujeres, que no provienen únicamente de distintas construcciones anatómicas, pero también de las condiciones culturales y sociales. El concepto de género no es universal, concretándose en cada sociedad, de acuerdo con los contextos de tiempo y espacio (Facio, Fries, 2005, p. 277). Tampoco es algo con que se nace, ni algo que se tiene. Como lo muestra Judith Butler (2007, pp. 263-275), es un acto performativo.

A pesar de que los estudios sobre la identidad del género vienen de la psicología y psiquiatría ${ }^{2}$, su aparición en las ciencias sociales le debe mucho a la

1 Antes de elección de Néstor Kirchner como presidente de la Nación, Cristina Fernández de Kirchner fue Senadora de la Nación Argentina (1995-1997, 2001-2005) y Diputada de la Nación Argentina (1997-2001).

2 El psicólogo Robert Stoller (1968) comenzó a utilizar el término "sexo" para describir los rasgos biológicos y "género" para escoger la cantidad de la feminidad y la masculinidad y la persona expuesta. La separación de estos términos le permitió a Stoller explicar el fenómeno de la transexualidad: el sexo y el género de los transexuales simplemente no coinciden. 
teoría feminista (Freud, 1994; Brickell, 2006; Lorber, Farrell, 1991). La distinción entre sexo y género fue usada por las investigadoras y investigadores feministas para enfatizar que muchas diferencias entre los hombres y las mujeres se producen socialmente y, por lo tanto, se las puede cambiar. La adopción de la perspectiva de género permite destacar los efectos de la construcción social de los géneros, sugiriendo nuevas formas de construir la masculinidad y la feminidad, sin basarlas en la discriminación (Inda, 2006, pp. 37-54). Entonces, el concepto de género debe ser relacionado con la emancipación y con la igualdad. Hablar sobre las cuestiones de género significa analizar los asuntos desde el punto de vista más amplio, más justo y más inclusivo. Por lo tanto, las reformas de género tienen que ser consideradas como reformas igualitarias, progresistas y antidiscriminatorias.

El objetivo principal del presente artículo es analizar las políticas de género -propuestas y agendas, legislación e iniciativas políticas- durante la presidencia de Cristina Fernández de Kirchner en Argentina. El análisis indicará tanto éxitos como fracasos de los programas, permitiendo una evaluación integral de la agenda kirchnerista de género. La investigación se ha llevado a cabo basándose en fuentes primarias (principalmente actos legislativos) y fuentes secundarias con análisis de tipo cualitativo.

Primero, se van a analizar los éxitos de la presidencia de Cristina Férnandez -en la legislación y en las cuestiones de la violencia de género. A continuación, se van a presentar los problemas y las derrotas de la agenda kirchnerista en el ámbito de género. En la conclusión se van a resumir los argumentos anteriores y se va a tratar de responder a la pregunta, si la presidencia de Cristina Fernández de Kirchner puede ser considerada como un paso adelante en materia de la igualdad de géneros, los derechos igualitarios y el empoderamiento de las mujeres argentinas.

\section{Los éxitos de la presidencia - las leyes igualitarias y de violencia de género}

\section{Las leyes igualitarias}

Desde el punto de vista de los derechos civiles, uno de los mayores logros de la presidencia de Cristina Fernández de Kirchner fue la adopción de la ley que permite a las personas homosexuales a contraer matrimonio en las mismas condiciones que las parejas heterosexuales. La ley de "matrimonio igualitario", promulgado en 2010, convirtió a Argentina en el primer país latinoamericano en legalizar los matrimonios homosexuales, algo que en los años 90 parecía impensable.

Hay que destacar que la promulgación de la ley tan auspiciosa para la igualdad sexual fue la culminación de un largo camino que se había iniciado más de cuatro décadas antes. Las primeras manifestaciones a favor de los derechos
Las políticas de género durante la presidencia de Cristina Fernández de Kirchner en Argentina (2007-2015)

Magdalena Lisińska 
Dossier América Latina: género y política de los homosexuales en Argentina empezaron a surgir a finales de los años 60 . En 1969 fue fundada la primera organización de derechos LGBT en Argentina - Nuestro Mundo (Mogroviejo, 2000, pp. 281-282). Los esfuerzos de los activistas fueron reprimidos durante la última dictadura militar 1976-1983 y pudieron resurgir sólo a finales de los años 80 y principios de la década de 1990 (Barrancos, 2014, p. 41). Una de las figuras más destacadas de este periodo fue Carlos Luis Jaúregui, un activista y primer presidente de la Comunidad Homosexual Argentina (CHA) entre 1984 y 1987. En 1990, la CHA propuso la ley de Matrimonio Civil con acceso para las parejas del mismo sexo, pero el proyecto fue rechazado (Historia del Matrimonio... s.f.). El movimiento LGBT logró la ley de unión civil que fue aprobada el 12 de diciembre de 2002 en la Ciudad Autónoma de Buenos Aires (Ley 1004, 2002). Fue un paso importante, pero insuficiente, ya que sólo incluyó la capital.

El proyecto de unión civil nacional fue rechazado por el parlamento en 2005 (Krasnow, 2012, p. 9). Los cambios de las leyes fueron posibles a partir de 2006, con el Congreso de la Nación dominado por la coalición kirchnerista Frente Para la Victoria. En 2008, el gobierno argentino puso en vigencia las pensiones por viudez para parejas homosexuales que puedan demostrar al menos cinco años de convivencia (Resolución 671/2008, 2008). Los trabajos sobre la ley de matrimonio igualitario empezaron en 2009 , coincidiendo en el tiempo con las acciones de amparo en la justicia, reclamando el derecho al matrimonio de parejas del mismo sexo. El 13 de noviembre de 2009, la jueza Gabriela Seijas del Fuero Contencioso Administrativo y Tributario en la Ciudad de Buenos Aires declaró inconstitucionales los artículos del Código Civil que prohíben la boda entre las personas del mismo sexo, permitiendo casarse a los peticionantes Alex Freyre y José María Di Bello. El casamiento tuvo lugar el 28 de diciembre de 2009 y fue el primer matrimonio civil entre personas del mismo sexo en Latinoamérica y el Caribe (Corrales, Pecheny, 2010, p. 435). En este contexto, el 5 de mayo de 2010 la Cámara de Diputados de la Nación sancionó el proyecto de matrimonio igualitario con 126 votos a favor, 110 en contra y 4 abstenciones. El 15 de julio de 2010, el Senado de la Nación aprobó la ley con 33 votos a favor, 27 en contra y 3 abstenciones (Honorable Cámara de Diputados de la Nación, 2010). La Ley Nacional $N^{\circ} 26.618$ modifica algunos artículos del Código Civil, otorgando los mismos derechos de matrimonio, seguridad social, adopción, herencia a las parejas heterosexuales y homosexuales (Ley 26.618, 2010). El documento fue promulgado por la presidenta Cristina Fernández de Kirchner el 21 de julio de 2010, con la presencia de los representantes de movimiento LGBT. En el discurso de promulgación, Fernández afirmó que aquel día los argentinos eran una sociedad más igualitaria que la semana anterior. "No hemos promulgado una ley, hemos promulgado una construcción social (...) que es transversal, diversa, plural y amplia y que no le pertenece a nadie, sino a quienes la construyeron: la sociedad" - dijo la presidenta (Díaz, 2014, pp. 123-125).

La otra ley muy importante que fue adoptada durante la presidencia de Cristina Fernández de Kirchner es la regulación de la identidad de género. 
La ley promulgada en 2012 permite a las personas transexuales, transgéneros y travestis registrarse legalmente con su nombre y género elegido. La importancia de la ley del 2012 es aún mayor porque hasta ese momento no existía ningún acto judicial que regulara el proceso de cambio de sexo en la Argentina. Entonces, la última palabra en la autorización de cirugías, terapias, cambios de nombre y de sexo registral pertenecía a los tribunales. Las personas trans tenían que pasar por procedimientos difíciles y humillantes, sin tener la garantía del fallo jurídico positivo. Cabe destacar que la primera decisión judicial en la que se reconoció el derecho a cambiar el sexo fue adoptada recién en 1997 (Caso N.N. 1997), pero hasta el 2010 los cambios legales fueron autorizados exclusivamente en casos de haber tenido la cirugía de reasignación genital previamente realizada, o cuando se expresara el compromiso de realizarla (Faji Neer, 2015, p. 354). Esto dio lugar a una situación patológica en la que el cambio formal del sexo requería una cirugía llevada a cabo en el extranjero, ya que tal procedimiento era ilegal en Argentina. La decisión del tribunal porteño en 2010, que autorizó el cambio del sexo de la famosa actriz Florencia de la V sin solicitar la confirmación médica (Farji Neer, 2014, p. 59), fue un impulso para las modificaciones de la ley nacional. Los trabajos sobre una nueva regulación comenzaron ya en 2010 y terminaron con la adopción de la ley a finales del 2011 y principios del 2012. Hay que destacar que las controversias por este proyecto eran menores que en el caso de matrimonio igualitario. El 2 de diciembre de 2011, la Cámara de Diputados de la Nación aprobó la ley por 167 votos a favor, 7 abstenciones y 17 en contra. Durante la votación en el Senado de la Nación, 55 senadores votaron a favor y sólo uno se abstuvo (Cano, Yacovino, 2014, p. 5). La ley 26.743 fue promulgada por la presidenta el 23 de mayo de 2012, siendo una de las regulaciones más liberales del mundo en materia de la identidad de género.

La regulación permite a cada persona que tenga como mínimo 18 años solicitar la rectificación registral de su sexo o un cambio de nombre de pila e imagen, basándose únicamente en la voluntad. Cabe destacar que la ley garantiza a toda persona el reconocimiento de su identidad de género, la libertad de desarrollar de su persona y el tratamiento de acuerdo con su identidad (Ley 26.743, 2012). Durante una ceremonia, Cristina Fernández de Kirchner entregó los primeros DNI bajo la nueva ley y dijo que había luchado mucho por esta ley, porque la igualdad es tan importante como la libertad. La presidenta tenía razón diciendo que, en cuanto a los derechos de las personas trans, no hay que hablar de tolerancia. Hay que hablar de igualdad, porque "la igualad es basamento del crecimiento de una cultura de toda una sociedad" (Casa Rosada, 2012).

La otra regulación que hay que mencionar al analizar la legislación de género en la Argentina es la ley de Fertilización Asistida, sancionada y promulgada en 2013. Hay que destacar que la regulación garantiza el acceso a los procedimientos y técnicas de reproducción médicamente asistida al grupo muy amplio de la sociedad, solamente basándose en el consentimien-
Las políticas de género durante la presidencia de Cristina Fernández de Kirchner en Argentina (2007-2015)

Magdalena Lisińska 
Dossier América Latina: género y política to informado (Ley 26.862, 2013). Es muy importante señalar que, según la ley, la fertilización asistida no es considerada como el tratamiento de una enfermedad, sino como un derecho universal de cada persona. Por lo tanto, la regulación no establece requisitos en forma de tener que presentar ningún tipo de diagnóstico ni certificación médica previa (Faji Neer, 2015, p. 352). Entonces, todas las personas mayores de edad tienen acceso a los procedimientos, abriendo la puerta tanto para las parejas homosexuales, como para las mujeres solteras que quisiesen tener hijos. Cuando promulgó la ley, la presidenta felicitó a todos que lucharon por la regulación, señalando que vivimos en un siglo en el cual el avance de la ciencia ha permitido introducir más inclusión y más derechos (Fernández de Kirchner, 2013). En 2015, Cristina Fernández inauguró el primer centro gratuito de fertilización asistida, ubicado en Córdoba (Télam, 2015).

\section{Las leyes de violencia de género}

En cuanto a las cuestiones de género, la sanción de regulaciones contra la violencia han sido uno de los mayores logros de los años de presidencia de Cristina Fernández de Kirchner. Según el informe de La Casa del Encuentro, una ONG que defiende los derechos de las argentinas, 1808 mujeres fueron asesinadas por violencia de género en Argentina entre 2008 y 2014, que se traduce a una muerte cada 30 horas (La Casa del Encuentro, 2014, p. 20). No cabe duda de que la violencia contra las mujeres viene de la discriminación sistémica, un fenómeno común en el mundo latinoamericano. Como señala la Convención sobre la Eliminación de Todas las Formas de Discriminación contra la Mujer, dicha discriminación tiene sus raíces en la cultura y tradición, manifestándose en hábitos, normas y estereotipos que excluyen a las mujeres de la esfera pública dominada por los varones (United Nations, 1979). Por lo tanto, violencia contra las mujeres no es solo un desafío criminal, sino también debe ser tratada como un problema de igualdad de género.

En 2002, Argentina ratificó el Protocolo de Palermo para prevenir, reprimir y sancionar la trata de personas, especialmente mujeres y niños, que complementa la Convención de las Naciones Unidas contra la Delincuencia Transnacional Organizada en el 2000 (United Nations, 2000). El resultado más importante de la ratificación, aunque retrasado, fue la sanción de la ley de trata de personas en 2008. La ley 26.364 diferenciaba la trata de personas mayores y menores de 18 años. Las penas eran de tres a seis años de prisión en caso de trata de mayores de 18 años, y de cuatro a 10 años por la trata de menores de 18 años. Si la víctima hubiese sido menor de 13 años, la pena podría haber sido aumentada de 6 a 15 años de prisión (Ley 26.364, 2008).

Aunque la regulación de la trata de personas fue un avance legal importante, las disposiciones de la ley, especialmente la distinción entre víctimas mayores y menores de edad causaba muchas controversias. Sobre todo, en el 
caso de los mayores de 18 años, el consentimiento de la víctima podía ser un elemento exculpable para el responsable de trata de personas. El debate por la modificación de la ley empezó en 2010, y el 31 de agosto de 2011 el Senado de la Nación sancionó por unanimidad la nueva regulación. Desgraciada e inexplicablemente, la Cámara de Diputados congeló el proyecto en la Comisión de Legislación Penal. La situación cambió 18 meses después, luego del veredicto en el caso judicial de Marita Verón, la mujer de 23 años secuestrada en 2002 con fines de trata de personas para la prostitución. En febrero de 2012, el caso hubiese sido llevado ante el tribunal, pero en diciembre todos los acusados fueron absueltos. El fallo generó enorme descontento y las olas de protestas en todo el país (Terán, 2012, pp. 1-2). Como reacción, la presidenta Cristina Fernández de Kirchner decidió convocar a sesiones extraordinarias de la Cámara de Diputados, para que la nueva ley fuese sancionada antes del fin de la sesión parlamentaria. Los diputados aprobaron por unanimidad la reforma, y la ley 26.842 fue promulgada el 26 de diciembre de 2012 (Serra, 2012). La nueva norma introdujo la definición de trata de personas, sin distinción de edad de la víctima, eliminando el consentimiento de la víctima como elemento excluyente de la responsabilidad criminal. Según la ley, la trata de personas es "el ofrecimiento, la captación, el traslado, la recepción o acogida de personas con fines de explotación, ya sea dentro del territorio nacional, como desde o hacia otros países" (Ley 26.842, 2012). Además, en su artículo 26, la ley endureció las penas por la trata de personas, castigando con prisión de cuatro a seis años al que promoviese o facilitase la prostitución de una persona, y con penas de 10 a 15 años de prisión en un gran número de casos, entre otros, si la víctima fuese menor de 18 años o mayor de 70 años, fuese una persona discapacitada, hubiese quedado embarazada, o las víctimas fuesen tres o más (Ley 26.842, 2012). La gran ventaja de la ley es que obliga al Estado brindar la asistencia a las víctimas de trata de personas, intentando dar un salto en la prevención. En 2015, la ley fue reglamentada a través del decreto $111 / 2015$ de la presidenta Cristina Fernández de Kirchner. La norma estableció la obligación de las fuerzas policiales y de seguridad a informar de inmediato la identificación de posibles víctimas al juez o fiscal encargados de la investigación, aumenta la ayuda a las víctimas de trata y promueve el funcionamiento del Consejo Federal para la Lucha contra la Trata y la Explotación de Personas y para la Protección y Asistencia a las Víctimas (Ghezzi, 2013, p. 92).

Hablando del problema de trata de personas, se debe mencionar otro proyecto, dirigido a profundizar la protección en esta materia: el decreto 936 de prohibición de avisos de oferta sexual, sancionado y promulgado por la presidenta Fernández de Kirchner en julio de 2011 (Decreto 936/2011, 2011). En 2014, el Congreso de la Nación empezó a trabajar sobre la ley que regulara las cuestiones de avisos sexuales. El proyecto fue aprobado por la Cámara de Diputados en noviembre de 2014, pero la regulación no ha sido adoptada hasta ahora. Según las asociaciones de defensa de los derechos civiles, el proyec-
Las políticas de género durante la presidencia de Cristina Fernández de Kirchner en Argentina (2007-2015)

Magdalena Lisińska 

América Latina: género y política to podría amenazar derechos constitucionales, como la libertad de expresión (Busaniche, 2014).

Además, los intentos legislativos de prevenir la violencia de género se manifestaron en otra norma - la ley 26.845 de protección integral de las mujeres. La ley fue votada durante la primera sesión del período legislativo en 2009 y promulgada el 1 de abril de 2009. Siendo una norma compleja y amplia, la ley define la violencia contra las mujeres como "toda conducta, acción u omisión que, de manera directa o indirecta, tanto en el ámbito público como en el privado, basada en una relación desigual de poder, afecte su vida, libertad, dignidad, integridad física, psicológica, sexual, económica o patrimonial, como así también su seguridad personal. Quedan comprendidas las perpetradas desde el Estado o por sus agentes”. Además, la ley señala la existencia de la violencia indirecta, entendida como "toda conducta, acción, omisión, disposición, criterio o práctica discriminatoria que ponga a la mujer en desventaja con respecto al varón" (Ley 26.485, 2009). Hay que destacar que en su artículo 5 la ley abarca de distintas formas de violencia contra las mujeres, presentando los cinco tipos básicos: 1) física, 2) psicológica, 3) sexual, 4) económica y patrimonial, 5) simbólica (Ley 26.485, 2009). Sancionando esta ley, Argentina se colocó entre los países que protegen las mujeres contra la violación de los derechos humanos tanto de la primera como de la segunda generación. En el plano institucional, el Consejo Nacional de las Mujeres, funcionando en Argentina desde 1991, fue definido como el organismo responsable de coordinación de las políticas públicas para efectivizar las disposiciones de la ley (Ley 26.485, 2009). Además, la norma contempla la creación del Observatorio de la Violencia contra las Mujeres, un Consejo Consultivo integrado por representantes de las organizaciones de la sociedad civil y del ámbito académico especializados, y establece las medidas en favor de las víctimas de la violencia, como la asistencia económica, legal, laboral, de salud. La sanción de la ley fue complementada con la aprobación del Decreto Reglamentario 1011/2010, firmado por la presidenta (Decreto 1011/2010, 2010). En pocas palabras, el objetivo principal de la norma era desarrollar un enfoque integral y complementario al problema de la violencia de las mujeres, así como crear la red de apoyo efectiva a las víctimas y la elaboración de estadísticas para evaluar el fenómeno.

\section{Las derrotas de la agenda kirchnerista}

A pesar de éxitos significativos en el campo legislativo, sería dudoso decir que la presidencia de Cristina Fernández de Kirchner fue una etapa totalmente progresista en cuanto al enfoque oficial de las cuestiones de género. El problema consiste en la ineficacia y falta de legislación, pero también, que parece más grave, en los errores conceptuales y la falta de comprensión de los asuntos básicos por parte de la presidenta. 


\section{La ineficacia de las leyes}

Uno de los problemas básicos de la agenda kirchnerista en cuanto a las cuestiones de género fue la falta de eficacia de las reformas introducidas. La aplicación efectiva de las disposiciones siempre es un elemento clave, sin el cual no se puede hablar del éxito de la agenda.

Los problemas con efectividad de la legislación fueron especialmente evidentes en el caso de la ley de protección integral de las mujeres del 2009. En 2015, a seis años de la sanción de la norma, fue publicado un informe del Equipo Latinoamericano de Justicia y Género (ELA) sobre el nivel de progreso de la implementación de la ley. Los autores del informe indican claramente, que no se podía hablar del éxito de la legislación. Primero, por falta de información oficial, no era posible medir la gravedad del problema de la violencia de género (Gheradi, 2015, pp. 21-24). Como destaca Natalia Gherardi, directora ejecutiva del Equipo de Justicia y Género, no había posibilidad de diseñar una política adecuada sin conocer los datos sobre la violencia en Argentina, tanto en el nivel nacional como en provincias (Carbajal, 2015). Tampoco se podía evaluar las iniciativas llevadas, ya que hasta 2017 no existió oficialmente el Plan Nacional de Acción para la Prevención, Asistencia y Erradicación de la Violencia contra las Mujeres. Cabe destacar que la elaboración, publicación, y implementación del Plan era un mandato de la Ley 26.485 (2009).

Además, muy preocupante en el contexto de dicha ley fue el comportamiento de la presidenta Cristina Fernández de Kirchner, que parecía estar muy distanciada de los temas relativos a las mujeres. La presidenta no había mencionado la ley de Protección Integral durante la apertura de sesiones parlamentarias en 2009, a pesar de que la legislación fue votada tres días después, en la primera sesión del período legislativo. Incluso después, la presidenta nunca la mencionó. Fernández de Kirchner tampoco reaccionó cuando, en junio de 2015, las organizaciones de los derechos humanos y los derechos de las mujeres dirigieron una carta a la presidenta del Consejo Nacional de las Mujeres, exigiendo la inmediata y amplia difusión pública del Plan Nacional de Acción para la Prevención, Asistencia y Erradicación de la Violencia contra las Mujeres (ELA, 2015). Debe destacar que el dicho Consejo había dependido directamente de la Presidencia, pero durante el kirchnerismo fue degradado bajo la órbita del Consejo de Coordinación de Políticas Sociales y lo dirigía una funcionaria sin formación en género (Stiegler, Gerber, 2009, p. 38).

\section{La falta de reformas}

Analizando las derrotas de la agenda kirchnerista de género, no sólo hay que destacar la ineficacia de las leyes, pero también la falta de las reformas clave para la igualdad de género.
Las políticas de género durante la presidencia de Cristina Fernández de Kirchner en Argentina (2007-2015)

Magdalena Lisińska 
Dossier América Latina: género y política
El problema que desde hace años se aplica a la Argentina, y que no se podía resolver durante la presidencia de Fernández de Kirchner, es la incompatibilidad de la ley del aborto a las realidades de la vida. El aborto no punible en Argentina está regulado por el artículo 86 del Código Penal del año 1921. Los incisos 1 y 2 establecen dos condiciones para el aborto: cuando la vida o la salud de la mujer está en peligro o cuando el embarazo es resultado de una "violación3 o de un atentado al pudor cometido sobre una mujer idiota o demente" (Ley 11.179, 1921). La legislación restrictiva, combinada con la falta de conciencia y de educación sexual dieron como resultado la emergencia de un problema social enorme, uno de los más graves peligros a la salud de las mujeres argentinas. Diversos informes, tanto internacionales como nacionales, confirman que el embarazo terminado en aborto clandestino, en condiciones inseguras, es la primera causa obstétrica directa de muerte materna en Argentina - el 23 \% entre los años 2007 y 2011 (Romero, Abalos, 2013). No cabe duda de que dicha legislación es socialmente perjudicial y sólo contribuye al sufrimiento de las mujeres que se quedaron embarazadas sin desearlo.

En realidad, Cristina Fernández de Kirchner nunca fue una entusiasta de liberalización de la ley del aborto -es algo que las activistas feministas no pueden perdonarle. Antes de ganar las elecciones de 2007, Fernández de Kirchner se declaró en contra del aborto. "Siempre me he definido en contra del aborto, aunque tampoco creo que nadie esté a favor del aborto. Mi postura siempre ha sido clara en ese sentido" - dijo (Peker, 2007). La presidenta guardó silencio cuando la ministra de salud de su primer gabinete, Graciela Ocaña, consideró el problema del aborto como "un tema del sistema penal", no de salud pública (Stiegler, Gerber 2009, p. 39). Además, Kirchner no emitió comentarios sobre un debate parlamentario de la despenalización del aborto en noviembre de 2011 (Gallo, 2011). El debate fracasó en la Cámara de Diputados menos de dos semanas después del inicio. Aunque en 2015 el ministro de salud del segundo gabinete de Kirchner, Daniel Gollán, anunció su intención de reiniciar un debate sobre el tema, fue desautorizado por el Jefe de Gabinete, Aníbal Fernández. La presidenta no hizo comentarios (BBC Mundo, 2015). Hay que recordar que en caso de la ley de aborto abstenerse de tomar una decisión es una decisión en sí misma. La falta de comprensión por parte de la presidenta mujer puede ser considerada como especialmente lamentable y preocupante.

\section{Problemas conceptuales y falta de comprensión}

La falta de entusiasmo de la presidenta en cuanto a varios proyectos relacionados con los asuntos de género fue una manifestación visible del estilo de

3 En marzo de 2012, la Suprema Corte de Justicia decidió que el aborto no es punible en violaciones cometidas sobre cualquier mujer y se lo puede realizar sin necesidad de recurrir a la justicia (Corte Suprema de la Justicia de la Nación, 2012). 
gobernar kirchnerista. Se puede resaltar una serie de síntomas que muestran baja sensibilidad feminista de Cristina Kirchner, sin la que no se puede hablar sobre un liderazgo conscientemente concentrado en las cuestiones de género.

Ya en la campaña electoral para la presidencia, Cristina Kirchner trataba de apelar a las mujeres que la llamaran por su primer apellido, Cristina Fernández, y pedía que usaran la forma femenina del cargo al que aspiraba: la presidenta. Además, en sus discursos, llamaba a las mujeres "hermanas de género", buscando empatía con sus congéneres” (Maffía, 2009, p. 16). Sin embargo, durante la visita a Francia, Kirchner pudo decir "no soy feminista, soy peronista", revelando su falta de sensibilidad de género e ignorancia en comprender el término "feminismo" (Diario Argentino, 2007).

Como señala Dora Barrancos, Cristina Kirchner fue muy ambigua y contradictoria en las cuestiones de género. El ambiente político en Argentina sigue siendo dominado por los varones, ya que las mujeres, cuando les toca mandar, suelen imitar a ellos (Di Marco, Barrancos, 2009). Lo mismo se aplicó a la presidencia de Cristina Fernández de Kirchner, que además tenía que enfrentar comparaciones con su marido, el expresidente Néstor Kirchner. A pesar de ser la mujer-presidenta, en su gabinete Cristina Fernández designó sólo tres mujeres ministras, de Ministerios de Desarrollo Social, Defensa y Salud. Ellas representaban la cuarta parte del total de ministerios. En cuanto a las mujeres en cargo de secretarías, fue el $22 \%$ de total y el $23 \%$ en cuanto a las subsecretarías (United Nations, 2008, p. 77). Además, Cristina Kirchner nunca habló sobre la necesidad de elevar el cupo femenino en el Congreso del 30 al 50 por ciento, que sería lógico, ya que las mujeres forman la mitad de la sociedad de la Argentina. Por lo tanto, es evidente que ni en su propio ambiente la presidenta tenía ambiciones de cambiar las relaciones de género y promover la equidad.

\section{Conclusiones}

En cuanto a las cuestiones de género, la presidencia de Cristina Fernández de Kirchner fue un período de cambios significativos para la Argentina. No cabe duda de que gracias a las reformas igualitarias, como el matrimonio homosexual, las leyes de identidad de género y de fertilización asistida, la Argentina se encuentra en el grupo de los países latinoamericanos más avanzados en igualdad de género, siendo incluso más avanzada que varios países de la Unión Europea, como Polonia, Eslovaquia, Rumania, Bulgaria, Letonia o Lituania. También son dignos de elogio los intentos legales de eliminar la violencia contra las mujeres.

Por otro lado, desgraciadamente, durante la presidencia de Cristina Kirchner no se puede hablar de cambio extenso del pensamiento sobre las cuestiones de género, especialmente entre los políticos. La falta de voluntad por cambiar la ley de aborto, una de las legislaciones más perjudiciales para las mujeres argentinas, tanto como la resistencia a la implementación de las dis-
Las políticas de género durante la presidencia de Cristina Fernández de Kirchner en Argentina (2007-2015)

Magdalena Lisińska 
Dossier América Latina: género y política

posiciones de la ley de protección integral, sirven como un ejemplo sobre esta situación preocupante. Lo que es aún más decepcionante, es que la primera mujer presidenta elegida en las elecciones generales en Argentina, no fuese suficientemente sensible a las cuestiones de género y no hiciese más para reducir la brecha entre los hombres y las mujeres. Cristina Fernández de Kirchner defendió los derechos de las minorías sexuales, pero no hizo lo suficiente para empoderar a las mujeres. Como la persona ejerciendo la función más elevada en el país, la presidenta hubiese podido servir como un ejemplo, introduciendo buenas prácticas en su ambiente político, como lo trataba de hacer Michelle Bachelet en Chile. Desgraciadamente, su estilo de ejercer el poder no fue nada diferente de los varones políticos en Argentina (Maffía, 2005).

No obstante, debe destacar que la opinión de Cristina sobre el aborto se ha modificado desde final de su presidencia. En agosto de 2018, como senadora nacional, Kirchner declaró que estaría a favor de la legalización del aborto en la votación en el Senado, explicando que no había alternativas y que la penalización no contribuía a la disminución de los abortos inseguros en Argentina. No obstante, la propuesta de aborto legal fue una vez más rechazada por cámara alta del Congreso en agosto de 2018 (Senado Argentina, 2008, p. 13). Argentina sigue siendo un país donde miles de mujeres son hospitalizadas o mueren por abortos inseguros cada año.

Concluyendo, hay que decir que las cuestiones de género en Argentina durante la presidencia de Cristina Fernández de Kirchner fueron un tema presente en el debate público y parlamentario. Se han introducido una serie de reformas igualitarias importantes, en línea con las tendencias presentes en los países desarrollados. Por otro lado, lamentablemente, la oportunidad de realizar cambios complejos y adaptar la legislación a las realidades de la vida no ha sido plenamente aprovechada. Los movimientos feministas, como el nacido en 2015 "Ni una menos", muestran que la violencia de género en Argentina es un problema público que necesita soluciones complejas, legislativas e implementadas por el Estado y sus instituciones responsables. La Argentina de hoy, bajo el gobierno de Mauricio Macri, sigue teniendo un largo camino para recorrer. Tiene que cambiar mucho para que se pueda decir que es un país realmente igualitario.

\section{Referencias bibliográficas}

Barrancos, D. (2014). Géneros y sexualidades disidentes en la Argentina: de la agencia por los derechos a la legislación positiva. Cuadernos Inter.c.a.mbio sobre Centroamérica y el Caribe, 11(2), pp. 17-46.

Busaniche, B. (2014). Las buenas intenciones y el camino a la censura. Fundación Vía Libre. Recuperado 18.02.2019 de https://www.vialibre.org.ar/2014/09/18/las-buenas-intenciones-y-elcamino-a-la-censura/ 
BBC Mundo (2.03.2015). Argentina: ley del aborto genera polémica en el gobierno de Cristina Fernández. Recuperado 18.02.2019 de http://www.bbc.com/mundo/ultimas_noticias/2015/03/150302_ultnot_ argentina_aborto_ministro_az

Brickell, C. (2006). The Sociological Construction of Gender and Sexuality. The Sociological Review, 54(1), pp. 87-113, DOI: https://doi.org/10.1111/j.1467-954X.2006.00603.x.

Butler, J. (2007). El género en disputa. Barcelona: Paidós.

Cano, J. E. y Yacovino, M. L. (2014). Identidad de género. Comparación crítica entre la ley española y la ley argentina, Trabajos de I Jornadas de Género y Diversidad Sexual. La Plata. Recuperado 16.02.2019 de http://sedici.unlp.edu.ar/bitstream/handle/10915/43055/Documento_completo. pdf? sequence $=1$

Carbajal, M. (2015). El tema estaba en la agenda pública pero no en la política. Página 12, 9 de junio. Recuperado 18.02.2019 de https://www.pagina12.com.ar/diario/sociedad/3-274481-2015-06-09. html

Casa Rosada (2.07.2012). Acto de entrega de DNI con reconocimiento del derecho a la identidad: Palabras de la Presidenta de la Nación. Recuperado 16.02.2019 de http://www.casarosada.gob. ar/informacion/archivo/25958-acto-de-entrega-de-dni-con-reconocimiento-del-derecho-a-laidentidad-palabras-de-la-presidenta-de-la-nacion

Caso N.N. (1997). La sentencia del 15 de mayo de 1997 del Juzgado de Primera Instancia en lo Civil y Comercial No 8 de Quilmes: “N.N.”. Identidad \& Diversidad. Recuperado17.02.2019 de https://identidadydiversidad.adc.org.ar/caso-n-n-1997/

Corrales, J. y Pecheny, M. (2010). The Politics of Sexuality in Latin America. A Reader on Lesbian, Gay, Bisexual, and Transgender Rights. Pittsburgh: University of Pittsburgh Press.

Corte Suprema de la Justicia de la Nación (13.03.2012). Causa "F. 259.XLVI 'F.A.L. s/ medida autosatisfactiva”. Recuperado 18.02.2019 de http://sjconsulta.csjn.gov.ar/sjconsulta/documentos/ verDocumentoByIdLinksJSP.html?idDocumento $=135171$

Di Marco, L. y Barrancos, D. (2009). Cristina es ambigua y contradictoria en cuestiones de género. La Nación, 5 de agosto. Recuperado 19.02.2019 de http://www.lanacion.com.ar/1158702cristina-es-ambigua-y-contradictoria-en-cuestiones-de-genero

Diario Argentino (3.02.2007). Cristina Kirchner viajará a Francia. Recuperado 19.02.2019 de https://www.diarioelargentino.com.ar/noticias/16166/cristina-kirchner-viajara-a-francia

Díaz, M. (2014). Anexo IX, Palabras de la Presidenta en acto de Promulgación Ley Matrimonio Igualitario. En La palabra y la acción: la máquina de enunciación K (pp. 123-125). Editorial EDUVIM.

Decreto 936/2011 (5.05.2011). Promuévese la erradicación de la difusión de mensajes e imágenes que estimulen o fomenten la explotación sexual. Recuperado 17.02.2019 de http://www.infoleg. gov.ar/infolegInternet/anexos/180000-184999/184133/norma.htm

Decreto 1011/2010 (2010, julio 19). Apruébase la reglamentación de la Ley No 26.485 que refiere a la protección integral para prevenir, sancionar y erradicar la violencia contra las mujeres en los ámbitos en que desarrollen sus relaciones interpersonales, disponible en: http://www.infoleg.gob. ar/infolegInternet/anexos/165000-169999/169478/norma.htm fecha de consulta: 18.02.2019.

Dirección Nacional Electoral (2007). Elecciones Presidenciales, resultados nacionales. Recuperado 15.02.2019 de https://recorriendo.elecciones.gob.ar/presidente2007.html\#/3/1

ELA, Equipo Latinoamericano de Justicia y Género (2015). Una treintena de organizaciones exige el Plan Nacional contra la violencia hacia las mujeres. Recuperado 18.02.2019 de http:// www.ela.org.ar/a2/index.cfm?muestra\&codcontenido=2160\&plcontampl=12\&aplicacion $=\operatorname{app} 187 \& \mathrm{cnl}=37 \&$ opc $=50$
Las políticas de género durante la presidencia de Cristina Fernández de Kirchner en Argentina (2007-2015)

Magdalena Lisińska 
Dossier América Latina: género y política
Facio, A. y Fries, L. (2005). Feminismo, género y patriarcado. Academia. Revista Sobre Enseñanza del Derecho de Buenos Aires, 3(6), pp. 268-273.

Farji Neer, A. (2014). Las tecnologías del cuerpo en el debate público. Análisis del debate parlamentario de la Ley de Identidad de Género argentina. Sexualidad, Salud y Sociedad - Revista Latinoamericana, (16), pp. 50-72, DOI: https://dx.doi.org/10.1590/S1984-64872014000100004.

Faji Neer, A. (2015). Cuerpo, derechos y salud integral: Análisis de los debates parlamentarios de las leyes de Identidad de Género y Fertilización Asistida (Argentina, 2011-2013). Salud Colectiva | Universidad Nacional de Lanús, 1(3), pp. 351-365, DOI: https://dx.doi.org/10.18294/ sc.2015.721.

Fernández de Kirchner, C. (2013). Felicitaciones a los que tanto lucharon por la ley de fertilización asistida. Recuperado 17.02.2019 de http://www.cfkargentina.com/felicitaciones-cristinaa-leyde-fertilizacion-asistida

Freud, S. (1994). The social construction of gender. Journal of Adult Development, 1(1), pp. 37-45.

Gallo, D. (2011). Aborto: debate sin aval presidencial. La Nación, 1 de noviembre. Recuperado de http://www.lanacion.com.ar/1419462-aborto-debate-sin-aval-presidencial

Gheradi, N. (2015). Claroscuros en las políticas contra la violencia de género. A cinco años de la sanción de la Ley 26.485 de Protección Integral contra la Violencia es tiempo de promover la rendición de cuentas. Recuperado 18.02.2019 de http://www.unfpa.org.ar/sitio/images/stories/pdf/2015. claroscuros_\%20politicas_contra_violencia_genero.pdf

Ghezi, A. C. (2013). Relevamiento normativo en materia de prevención y sanción del delito de trata de personas y de organismos estatales articulados. En Z. Gatti et al., Trata de personas. Políticas del Estado para su prevención y sanción (pp. 47-121). Buenos Aires: Infojus.

Historia del Matrimonio LGTB en Argentina (s.f), Organización a la comunidad LGBT Argentina. Recuperado 16.02.2019 de http://www.cha.org.ar/historia-del-matrimonio-lgtb-en-argentina/

Honorable Cámara de Diputados de la Nación (2010). Votación, Ley 26.618 Matrimonio Civil. Recuperado 16.02.2019 de http:/www1.hcdn.gov.ar/dependencias/dselectronicos/ actas/2010/1280E04_01_R07.pdf

Inda, N. (2006). La perspectiva de género en investigaciones sociales. En C. Verschuur y F. Hainard (Eds.), Des brèches dans la ville. Organisations urbaines, environnement et transformations des rapports de genre (pp. 37-54). Actes du colloque de l'IUED. Genève: Graduate Institute Publications.

Krasnow, A. N. (2012). El nuevo modelo de matrimonio civil en el derecho argentino. Revista de Derecho Privado, 22(1), pp. 5-39.

La Casa del Encuentro (2014). Observatorio de Femicidios en Argentina "Adriana Marisel Zambrano". Recuperado 17.02.2019 de http://www.seguridadciudadana.info/docs/Observatorio \%20de \%20Femicidios \%20en \%20Argentina2014.pptx \%20 \%281 \%29.pdf

Ley 1004 (12.12.2002). Unión civil. Recuperado 16.02.2019 de https://www.buenosaires.gob.ar/ sites/gcaba/files/ley1004.pdf

Ley 11.179 (29.10.1921). Código Penal de la Nación. Recuperado 18.02 .2019 de http://www. infoleg.gov.ar/infolegInternet/anexos/15000-19999/16546/norma.htm

Ley 26.364 (9.04.2008). Prevención y sanción de la trata de personas y asistencia a sus victimas. Disposiciones Generales. Derechos de las Víctimas. Disposiciones Penales y Procesales. Disposiciones Finales. Recuperado 17.02.2019 de http://www.infoleg.gov.ar/infolegInternet/ anexos/140000-144999/140100/norma.htm 
Ley 26.485 (11.03.2009). La ley de protección integral de las mujeres. Ley de protección integral para prevenir, sancionar y erradicar la violencia contra las mujeres en los ámbitos en que desarrollen sus relaciones interpersonales. Recuperado 18.02.2019 de http://www.infoleg.gov.ar/infolegInternet/ anexos/150000-154999/152155/norma.htm

Ley 26.618 (15.07.2010). Matrimonio Civil. Código Civil. Modificación. Disponible en: http:// www.infoleg.gov.ar/infolegInternet/anexos/165000-169999/169608/norma.htm, fecha de consulta: 16.02.2019.

Ley 26.743 (9.05.2012). Identidad de género. Establécese el derecho a la identidad de género de las personas. Recuperado 16.02.2019 de http://servicios.infoleg.gob.ar/infolegInternet/ anexos/195000-199999/197860/norma.htm

Ley 26.842 (19.12.2012). Prevención y sanción de la trata de personas y asistencia a sus víctimas. Código Penal, Código Procesal Penal y Ley No 26.364. Modificaciones. Recuperado 17.02.2019 de http://infoleg. mecon.gov.ar/infolegInternet/anexos/205000-209999/206554/norma.htm

Ley 26.862 (5.06.2013). Reproducción Medicamente Asistida. Acceso integral a los procedimientos y técnicas médico-asistenciales de reproducción médicamente asistida. Recuperado 16.02.2019 de http://www.infoleg.gob.ar/infolegInternet/anexos/215000-219999/216700/norma.htm

Lorber, J. y Farrell, S. A. (Eds.). (1991). The social construction of gender. Newbury Park, CA: Sage Publications.

Maffía, D. (2009). La agenda de género en el Gobierno de Cristina Kirchner. Ponencia presentada en el seminario Género en el Poder: el Chile de Michelle Bachelet, Observatorio de Género y Equidad, Santiago de Chile.

Maffía, D. (2005). Cristina Kirchner quiere ser Perón. La Nación, 25 de noviembre. Recuperado 19.02.2019 de https://www.lanacion.com.ar/759568-cristina-kirchner-quiere-ser-peron-dicediana-maffia

Mogroviejo, N. (2000). Un amor que se atrevió a decir su nombre: la lucha de las lesbianas y su relación con los movimientos homosexual y feminista en América Latina. Madrid: Plaza y Valdés.

Peker, L. (2007). Una mujer, muchas mujeres. Página 12, 2 de noviembre. Recuperado 18.02.2019 de https://www.pagina12.com.ar/diario/suplementos/las12/13-3689-2007-11-04.html

Resolución 671/2008 (19.10.2008). Declárase a los convivientes del mismo sexo incluidos en los alcances del artículo 53 de la Ley $N^{\circ} 24.241$, como parientes con derecho a la pensión por fallecimiento del jubilado, del beneficiario de retiro por invalidez o del afiliado en actividad del Régimen Previsional Público o del Régimen de Capitalización. Recuperado 16.02.2019 http://servicios.infoleg.gob.ar/ infolegInternet/anexos/140000-144999/143820/norma.htm

Romero, M., Abalos, E., Ramos, S. (2013). La situación de la mortalidad materna en Argentina y el Objetivo de Desarrollo del Milenio 5. Observatorio del Salud Sexual y Reproductiva. Recuperado 18.02.2019 de http://www.ossyr.org.ar/pdf/hojas_informativas/hoja_8.pdf

Senado Argentina (2008). El Senado trata el proyecto de interrupción voluntaria del embarazo. Senado de la Nación. Recuperado 19.02.2019 de http://www.senado.gov.ar/bundles/senadomicrositios/pdf/despenalizacion-aborto/comunicados.pdf

Serra, L. (2012). Tras 18 meses, se aprobó la ley contra la trata de personas, La Nación, 20 de diciembre. Recuperado 17.02.2019 de http://www.lanacion.com.ar/1538731-tras-18-meses-seaprobo-la-ley-contra-la-trata-de-personas

Stiegler, B. y Gerber, E. (2009). Género y Poder. El significado del género en los más altos cargos políticos: los casos de Alemania, Chile, Argentina y España. Santiago: Friedrich-Ebert-Stiftung.
Las políticas de género durante la presidencia de Cristina Fernández de Kirchner en Argentina (2007-2015)

Magdalena Lisińska 
Dossier América Latina: género y política
Stoller, R. (1968). Sex and Gender: On the Development of Masculinity and Femininity. New York: Science House.

Télam (20.03.2015). La Presidenta inauguró un centro gratuito de fertilización asistida en Córdoba. Recuperado 17.02.2019 de http://www.telam.com.ar/notas/201503/98774-cristinacentro-gratuito-fertilizacion-asistida-cordoba.html

Terán, K. (2017). Argentina’s Fight for the End of Human Trafficking. Council on Hemispheric Affairs. Recuperado 17.02.2019 de http://www.coha.org/wp-content/uploads/2017/02/Humantraffickingargentina-215-2.pdf

United Nations (1979). Convention on the Elimination of All Forms of Discrimination Against Women, New York, 18 December. Recuperado 17.02.2019 de https://treaties.un.org/doc/ Treaties/1981/09/19810903\%2005-18 \%20AM/Ch_IV_8p.pdf

United Nations (2000). Convention against Transnational Organized Crime, New York, 15 November. Recuperado 17.02.2019 de https://treaties.un.org/doc/Treaties/2000/11/20001115 \%2011-11 \%20AM/Ch_XVIII_12p.pdf

United Nations (2008). Consideration of reports submitted by States parties under article 18 of the Convention on the Elimination of All Forms of Discrimination Against Women, Sixth Periodic Report of States Parties. Argentina, CEDAW/C/ARG/6. Recuperado 19.02.2019 de http://www2. ohchr.org/english/bodies/cedaw/docs/AdvanceVersions/CEDAW-C-ARG-6_sp.pdf

Vitale, M. A. y Maizels, A. (2011). El discurso electoral de Cristina Fernández de Kirchner (2007): un caso de ethos híbrido no convergente. Linguagem em (Dis)curso, 11(2), pp. 337-360, DOI: https://dx.doi.org/10.1590/S1518-76322011000200007. 